\title{
ON FAMILIES OF CAUCHY TRANSFORMS AND BMOA
}

\author{
YUSUF ABU MUHANNA and EL-BACHIR YALLAOUI
}

(Received 7 December 1998; revised 23 October 1999)

Communicated by P. C. Fenton

\begin{abstract}
In this paper we prove a number of results on Cauchy transforms of generalized type given by Borel measures supported on the class of analytic functions mapping the unit disc into the unit disk. We also give a BMOA characterization using these families.
\end{abstract}

2000 Mathematics subject classification: primary: 30E20; secondary: 30D99.

Keywords and phrases: Cauchy transforms, BMOA.

\section{Introduction}

Let $\Delta=\{z \in \mathbb{C}:|z|<1\}, \Gamma=\{x:|x|=1\}$ and let $\mathscr{M}$ denote the sets of complex-valued Borel measures on $\Gamma$.

For $z \in \Delta$ and $\alpha \geq 0$, let $F_{\alpha}$ denote the family of functions $f$ for which there exists a measure $\mu \in \mathscr{M}$ such that

$$
f(z)= \begin{cases}\int_{\Gamma} \frac{1}{(1-\bar{x} z)^{\alpha}} d \mu(x) & \text { for } \quad \alpha>0 \\ \int_{\Gamma} \log \frac{1}{1-\bar{x} z} d \mu(x)+f(0) & \text { for } \quad \alpha=0 .\end{cases}
$$

These families are known as families of Cauchy transforms and have been well studied in $[4,3,8,10]$.

The class $F_{\alpha}$ is a Banach space with respect to the norm

$$
\|f\|_{F_{\alpha}}= \begin{cases}\inf \|\mu\| & \text { for } \quad \alpha>0 \\ \inf \|\mu\|+|f(0)| & \text { for } \quad \alpha=0,\end{cases}
$$

(C) 2000 Australian Mathematical Society 0263-6115/2000 $\$ A 2.00+0.00$ 
where $\mu$ varies over all measures in $\mathscr{M}$ for which (1.1) holds and where $\|\mu\|$ denotes the total variation norm of $\mu$.

It is known from $[5,6]$ that

- $F_{\alpha} \subset F_{\beta}$, whenever $0 \leq \alpha<\beta$.

- $F_{\alpha}$ is Möbius invariant for $\alpha \geq 0$.

- $f \in F_{\alpha}$ if and only if $f^{\prime} \in F_{1+\alpha}$.

- $\left\|f^{\prime}\right\|_{F_{1+\alpha}} \leq k\|f\|_{F_{\alpha}}$ for some $k>0$.

We now define generalized families of Cauchy transforms by extending $\Gamma$ and $\mathscr{M}$. We extend $\Gamma$ to $B$, the set of analytic selfmaps $\phi$ mapping the open unit disc $\Delta$ into itself and such that $\phi(0)=0$. We also extend $\mathscr{M}$ to $\mathscr{N}$, the sets of complex-valued Borel measures on $B . B$ is equipped with the topology of uniform convergence on compact subsets. Here $\mathscr{M}$ is equivalent to the subset of $\mathscr{N}$ consisting of all those measures supported on the set $\{x z:|x|=1\}$.

For $z \in \Delta$ and $\alpha \geq 0$, let $A_{\alpha}$ denote the family of functions $f$ for which there exists a measure $\mu \in \mathscr{N}$ such that

$$
f(z)= \begin{cases}\int_{B} \frac{1}{(1-\phi(z))^{\alpha}} d \mu(\phi) & \text { for } \quad \alpha>0 \\ \int_{B} \log \frac{1}{1-\phi(z)} d \mu(\phi)+f(0) & \text { for } \quad \alpha=0 .\end{cases}
$$

The class $A_{\alpha}$ is a Banach space with respect to the norm

$$
\|f\|_{A_{\alpha}}= \begin{cases}\inf \|\mu\| & \text { for } \quad \alpha>0 \\ \inf \|\mu\|+|f(0)| & \text { for } \quad \alpha=0\end{cases}
$$

where $\mu$ varies over all measures in $\mathscr{N}$.

Clearly the classes $F_{\alpha}$ are subsets of $A_{\alpha}$ when the measures $\mu$ in (1.3) are in $\mathscr{M}$. Furthermore, for $f \in F_{\alpha},\|f\|_{F_{\alpha}} \geq\|f\|_{A_{\alpha}}$. It is also known from [1] that for $\alpha \geq 1$, $F_{\alpha}=A_{\alpha}$.

We show in this paper that for $0 \leq \alpha<\beta$,

$$
A_{\alpha} \subset A_{\beta} \quad \text { and } \quad\|f\|_{A_{\beta}} \leq\|f\|_{A_{\alpha}} .
$$

This generalizes similar results for $F_{\alpha}$ (see $[7,9]$ ).

We also show that $A_{0}=\mathrm{BMOA}$ and that the norm $\|\cdot\|_{A_{0}}$ is equivalent to well known BMO norms. Furthermore, we show that, for all $n \geq 1$ and $\alpha \geq 0$

$$
\left\|z^{n}\right\|_{A_{\alpha}} \leq k
$$

where the constant $k$ is independent of $n$ and $\alpha$. 


\section{The classes $A_{\alpha}$}

In this section we establish for $0 \leq \alpha<\beta$ the relationship between $A_{\alpha}$ and $A_{\beta}$ as well as their respective norms.

THEOREM 2.1. If $0 \leq \alpha<\beta$, then $A_{\alpha} \subset A_{\beta}$ and $\|f\|_{A_{\beta}} \leq\|f\|_{A_{\alpha}}$.

PROOF. Note that since $A_{\alpha}=F_{\alpha}$ for $\alpha \geq 1$, [1], and for $0<\alpha<\beta, F_{\alpha} \subset F_{\beta}$ and $\|f\|_{F_{\beta}} \leq\|f\|_{F_{\alpha}}$ [7], then all we have to prove is the case $0 \leq \alpha<\beta<1$.

(i) Let $f \in A_{\alpha}$, where $0<\alpha<\beta$, then we can write

$$
f(z)=\int_{B} \frac{1}{(1-\psi(z))^{\alpha}} d \mu(\psi) .
$$

Since $1 /(1-z)^{\alpha} \in F_{\alpha} \subset F_{\beta}$, we can write

$$
\frac{1}{(1-z)^{\alpha}}=\int_{\Gamma} \frac{1}{(1-\bar{x} z)^{\beta}} d \nu(x)
$$

and

$$
\left\|\frac{1}{(1-z)^{\alpha}}\right\|_{F_{\beta}} \leq\left\|\frac{1}{(1-z)^{\alpha}}\right\|_{F_{\alpha}}=1 .
$$

Now by replacing $z$ in (2.2) by $\psi(z)$ and putting the result in (2.1) we get

$$
f(z)=\int_{B} \int_{\Gamma} \frac{1}{(1-\bar{x} \psi(z))^{\beta}} d \nu(x) d \mu(\psi) .
$$

Suppose without loss of generality that $v$ is a positive measure and let

$$
g_{n}(z)=\sum_{k=1}^{n} \frac{\nu_{k}}{\left(1-\bar{x}_{k} z\right)^{\beta}} .
$$

Then by (2.1)

$$
\int_{B} g_{n}(\psi) d \mu(\psi)
$$

converges locally uniformly to

$$
f(z)=\int_{B} \int_{\Gamma} \frac{1}{(1-\bar{x} \psi(z))^{\beta}} d v(x) d \mu(\psi) .
$$

Let $\eta_{n}(\psi)=\sum_{k=1}^{n} v_{k} \mu(\psi)$, then

$$
\int_{B} g_{n}(\psi) d \mu(\psi)=\int_{B} \frac{1}{(1-\psi)^{\beta}} d \eta_{n}(\psi),
$$


where $\left\|\eta_{n}\right\| \leq\|v\|\|\mu\|$ for all $n$. Hence by compactness, there exists a measure $\sigma$ such that

$$
f(z)=\int_{B} \frac{1}{(1-\psi(z))^{\beta}} d \sigma(\psi),
$$

which shows that $f \in A_{\beta}$. Furthermore, $\|\sigma\| \leq\|v\|\|\mu\|$. Consequently,

$$
\|f\|_{A_{\beta}} \leq\|\sigma\| \leq\|\mu\|\|\nu\| \quad \text { for all } \mu .
$$

However, since $\mu$ and $v$ are arbitrary measures that give (2.1) and (2.2), then

$$
\|f\|_{A_{\beta}} \leq \inf \{\|\mu\|\} \inf \{\|v\|\} .
$$

Hence by (2.3)

$$
\|f\|_{A_{\beta}} \leq\|f\|_{A_{\alpha}} \text {. }
$$

(ii) Now let $f \in A_{0}$. We want to show that $f \in A_{\alpha}$ for any $\alpha>0$. By the definition,

$$
f(z)=\int_{B} \log \frac{1}{1-\phi(z)} d \mu(\phi)+f(0)
$$

Since $\log (1-z)^{-1} \in F_{0} \subset F_{\alpha}$ see [5], then

$$
\log \frac{1}{1-z}=\int_{\Gamma} \frac{1}{(1-\bar{x} z)^{\alpha}} d v(x)
$$

where $v$ depends only on $\alpha$. Hence (2.6) becomes

$$
f(z)=\int_{B} \int_{\Gamma} \frac{1}{(1-\bar{x} \phi(z))^{\alpha}} d v(x) d \mu(\phi)+f(0),
$$

where the integral in (2.7) looks exactly like the one in (2.3) with $\alpha$ replacing $\beta$. Hence using an argument similar to the one in (i) we get that

$$
f(z)=\int_{B} \int_{\Gamma} \frac{1}{(1-\psi(z))^{\alpha}} d \sigma(\psi)+f(0)
$$

which shows that $f \in A_{\alpha}$. Furthermore, $\|\sigma\| \leq\|v\|\|\mu\|$ and hence

$$
\|f\|_{A_{\alpha}} \leq \inf \{\|\mu\|\}+|f(0)|=\|f\|_{A_{0}} .
$$




\section{Characterization of $A_{0}$}

It is known from [2, page 248] that $\phi \in \mathrm{BMO}$ if and only if there exist functions $\phi_{1}$ and $\phi_{2}$ in $L^{\infty}$ such that

$$
\phi=\phi_{1}+\tilde{\phi}_{2}+\alpha,
$$

where both $\left\|\phi_{1}\right\|_{\infty}$ and $\left\|\phi_{2}\right\|_{\infty}$ are less than $C\|\phi\|_{\star}, C$ is a constant and \|\|$_{\star}$ is the classical BMO norm.

Consequently $f \in \mathrm{BMOA}$ if and only if there are analytic functions $f_{1}$ and $f_{2}$ such that

$$
f=f_{1}+f_{2}+\alpha
$$

where $\left\|\operatorname{Re} f_{1}\right\|_{\infty} \leq C$ and $\left\|\operatorname{Im} f_{2}\right\|_{\infty} \leq C$. If we define on BMOA the norm

$$
\|f\|^{*}=\inf \left\{\left\|\operatorname{Re} f_{1}\right\|_{\infty}+\left\|\operatorname{Im} f_{2}\right\|_{\infty}: f=f_{1}+f_{2}+\alpha\right\},
$$

then by [2], the norms $\|f\|^{\star}$ and $\|f\|_{\star}$ are equivalent.

Now we have the following proposition which establishes a set equality between $A_{0}$ and BMOA and thus gives a BMOA characterization using generalized families of Cauchy transforms.

THEOREM 3.1. $A_{0}=$ BMOA.

PROOF. Suppose that $f \in A_{0}$, then according to (1.3) there exists a measure $\mu \in \mathscr{N}$ such that

$$
f(z)=\int_{B} \log \frac{1}{1-\phi(z)} d \mu(\phi)+f(0) .
$$

Assume without loss of generality that $\mu$ is a probability measure. Then $f$ is subordinate to $\log (1-z)^{-1}+f(0)$ and consequently by (3.1) $f \in$ BMOA. The proof of the other inclusion follows from (3.1) and subordination.

THEOREM 3.2. The norms $\|\cdot\|_{\star}$ and $\|\cdot\|_{A_{0}}$ are equivalent, that is, there exist positive constants $c_{1}$ and $c_{2}$ such that

$$
c_{1}\|f\|_{\star} \leq\|f\|_{A_{0}} \leq c_{2}\|f\|_{\star} .
$$

PROOF. Suppose $f \in$ BMOA, then $f$ can be decomposed as in (3.1). Let $d_{1}$ denote $\left\|\operatorname{Re} f_{1}\right\|_{\infty}$ and $d_{2}$ denote $\left\|\operatorname{Im} f_{2}\right\|_{\infty}$. Then

$$
\left|\frac{\pi}{2 d_{1}} \operatorname{Im} i f_{1}(z)\right| \leq \frac{\pi}{2}
$$


and

$$
\left|\frac{\pi}{2 d_{2}} \operatorname{Im} f_{2}(z)\right| \leq \frac{\pi}{2}
$$

for all $z \in \Delta$. Consequently, by subordination

$$
\begin{aligned}
\text { if } & (z)=\frac{2 d_{1}}{\pi}\left(\log \frac{1}{1-\phi(z)}-\log \frac{1}{1+\phi(z)}\right)+i f_{1}(0) \\
f_{2}(z) & =\frac{2 d_{2}}{\pi}\left(\log \frac{1}{1-\psi(z)}-\log \frac{1}{1+\psi(z)}\right)+f_{2}(0)
\end{aligned}
$$

for all $z \in \Delta$ and $\phi, \psi \in B$. Therefore,

$$
\|f\|_{A_{0}} \leq \frac{4}{\pi}\left(d_{1}+d_{2}\right),
$$

and hence

$$
\|f\|_{A_{0}} \leq \frac{4}{\pi}\|f\|^{*} \leq c_{2}\|f\|_{*}
$$

which gives the right inequality in (3.4).

Next, we show that the inequality (3.14) holds. Let us write $f$ as in (1.2) and assume without loss of generality that $\mu$ is a positive measure. Then

$$
|\operatorname{Im} f(z)| \leq c\|\mu\|,
$$

where $c>1$. Thus

$$
|\operatorname{Im} f(z)| \leq c\|f\|_{A_{0}}
$$

and since

$$
\|\operatorname{Im} f\|_{\infty} \geq\|\operatorname{Im} f\|_{\star}
$$

we have

$$
\|f\|_{\star} \leq k_{1}\|\operatorname{Im} f\|_{\star} \leq k_{2}\|f\|_{A_{0}}=\frac{1}{c_{1}}\|f\|_{A_{0}},
$$

where $c_{1}=1 / k_{2}$ and the left inequality in (3.14) follows from [2, page 235].

THEOREM 3.3. $\left\|z^{n}\right\|_{A_{\alpha}} \leq k$ for $n \geq 1$.

PROOF. It is enough to show that $\left\|z^{n}\right\|_{A_{0}} \leq k$ for $n \geq 1$. Since we have shown that $\|\cdot\|_{*}$ and $\|\cdot\|_{A_{0}}$ are equivalent, let us approximate $\left\|z^{n}\right\|_{*}$. It is known (see [2, page 240]) that

$$
\|g\|_{\star}^{2} \approx \sup _{\psi} \iint_{\Delta}|\nabla g|^{2}\left(1-|z|^{2}\right)\left|\psi^{\prime}(z)\right| d A
$$


where $\psi(z)=\left(z+z_{0}\right) /\left(1+\overline{z_{0}} z\right)$ is a Möbius transformation. Replace $g$ in (3.15) by $z^{n}$ and $|z|$ by $r$ to get

$$
\begin{aligned}
I & =\iint_{\Delta}\left|\nabla\left(z^{n}\right)\right|^{2}\left(1-r^{2}\right)\left|\psi^{\prime}(z)\right| d A \\
& \leq \iint_{\Delta} n^{2} r^{2 n-2}\left(1-r^{2}\right)\left|\psi^{\prime}(z)\right| d A \\
& \leq \int_{0}^{2 \pi} \int_{0}^{1} n^{2} r^{2 n-2}\left(1-r^{2}\right)\left|\psi^{\prime}(z)\right| d r d \theta \\
& \leq 2 \pi n^{2} \int_{0}^{1} r^{2 n-2}(1-r) d r, \quad \text { because } \int_{0}^{2 \pi}\left|\psi^{\prime}(z)\right| d \theta \leq 2 \pi \\
& \leq 2 \pi n^{2} \int_{0}^{1}\left(r^{2 n-2}-r^{2 n}\right) d r=\frac{4 \pi n^{2}}{\left(4 n^{2}-1\right)} \leq \frac{4 \pi}{3}
\end{aligned}
$$

which gives us the desired result and completes the proof.

The following corollary is a direct consequence of the previous theorem.

COROLLARY 3.4. If $f(z)=\sum_{n=0}^{\infty} a_{n} z^{n}$ is analytic and if $\sum_{n=1}^{\infty}\left|a_{n}\right|<\infty$, then $f \in A_{\alpha}$ for all $\alpha \geq 0$.

PROOF. It is sufficient to prove that $f \in A_{0}$ since $A_{0} \subset A_{\alpha}$. To show that $f \in A_{0}$ all we have to show is that the norm $\|f(z)\|_{A_{0}}$ is bounded.

$$
\|f(z)\|_{A_{0}}=\left\|\sum_{n=0}^{\infty} a_{n} z^{n}\right\|_{A_{0}} \leq \sum_{n=0}^{\infty}\left|a_{n}\right|\left\|z^{n}\right\|_{A_{0}} \leq \frac{4 \pi}{3} \sum_{n=0}^{\infty}\left|a_{n}\right|<\infty .
$$

\section{Acknowledgement}

The authors would like to thank the referee for the many useful comments.

\section{References}

[1] D. A. Brannan, J. G. Clunie and W. E. Kirwan, 'On the coefficient problem for functions of bounded boundary rotation', Ann. Acad. Sci. Finn. Ser. Al Math. Dissertationes 523 (1973).

[2] J. Garnett, Bounded analytic functions (Academic Press, New York, 1980).

[3] D. J. Hallenbeck, T. H. MacGregor and K. Samotij, 'Fractional Cauchy transforms, inner functions and multipliers', Proc. London Math. Soc. (3) 72 (1996), 157-187.

[4] D. J. Hallenbeck and K. Samotij, 'On Cauchy integrals of logarithmic potentials and their multipliers', J. Math. Anal. Appl. 174 (1993), 614-634. 
[5] R. A. Hibschweiler and T. H. MacGregor, 'Closure properties of families of Cauchy-Stieltjes transforms', Proc. Amer. Math. Soc. 105 (1989), 615-621.

[6] — - 'Multipliers of families of Cauchy-Stieltjes transforms', Trans. Amer. Math. Soc. 331 (1992), 377-394.

[7] R. A. Hibschweiler and E. Nordgren, 'Cauchy transforms of measures and weighted shift operators on the disk algebra', Rocky Mount. J. Math. 26 (1996), 627-653.

[8] S. V. Hruscev and S. A. Vinogradov, 'Inner functions and multipliers of Cauchy type integrals', Arkiv Mat. 19 (1981), 23-42.

[9] T. H. MacGregor, 'Analytic and univalent functions with integral representations involving complex measure', Indiana Univ. Math. J. 36 (1987), 109-130.

[10] S. A. Vinogradov, 'Properties of multipliers of Cauchy-Stieltjes integrals and some factorization problems for analytic functions', Amer. Math. Soc. Trans. 115 (1980), 1-32.

Department of Mathematics

American University of Sharjha

PO Box 2666

Sharjha

United Arab Emirates

e-mail: ymuhanna@aus.ac.ae
Department of Mathematics and Statistics Sultan Qaboos University PO Box 36

Al-Khod P.C. 123

Muscat

Sultanate of Oman

e-mail: bachiry@squ.edu.om 